\title{
Cuidado de enfermería perdido percibido por madres de pacientes pediátricos
}

\author{
hospitalizados
}

\author{
Ada Lizette González-Sánchez ${ }^{1}$; ; María Guadalupe Moreno-Monsiváis ${ }^{*} \mathbb{D}$; Sofía Cheverría-Rivera ${ }^{3}$
}

\begin{abstract}
RESUMEN
Introducción: El cuidado de enfermería perdido se define como el cuidado requerido por el paciente que se omite (parcial o totalmente) o se retrasa significativamente. En la población pediátrica es relevante identificarlo dada la vulnerabilidad de los niños, quienes pueden no ser capaces de expresar o demandar la atención necesaria. Objetivo: Identificar el cuidado de enfermería perdido y su relación con las razones en función de la percepción de madres. Metodología: Estudio descriptivo correlacional. Participaron 162 madres de pacientes pediátricos de un hospital público. Los datos se recolectaron con el instrumento MISSCARE, para el análisis se utilizó estadística descriptiva e inferencial. Resultados: Los cuidados que se pierden principalmente son la planificación del alta y educación al paciente, seguido de cuidado básico como ayudar o realizar el lavado bucal y de manos (82.1\%), verificar que la comida esté a una temperatura adecuada (62.1\%) y acercar la comida y verificar la dieta antes de que la coman (61.1\%). Los cuidados se pierden en primer orden por razones de recursos humanos. Destacó el aumento inesperado en el volumen de pacientes y/o carga de trabajo (47\%) y por situaciones de urgencia que demandan mayor atención y generan poco tiempo para atender a otros pacientes (34.5\%). Conclusiones: Se identificó cuidado perdido en el paciente pediátrico hospitalizado y se atribuye principalmente a razones de déficit de recursos humanos. Esta información es relevante de considerar por los gestores para fortalecer el cuidado de enfermería en la población pediátrica.
\end{abstract}

Palabras clave: Enfermería; Cuidado; Paciente; Madre. (DeCS).

${ }^{1} \mathrm{MSc}$, Enfermera Especialista, Hospital Regional de Alta Especialidad del Bajío

${ }^{* 2} \mathrm{PhD}$, Investigadora, Profesor de Tiempo Completo, Facultad de Enfermería. Universidad Autónoma de Nuevo León.

${ }^{3} \mathrm{MSc}$, Investigadora, Profesor de Tiempo Completo, Facultad de Enfermería y Nutrición, Universidad Autónoma de San Luis Potosí.

*Autor para correspondencia: mgmoreno@hotmail.com

Recibido: $19 / 01 / 2020$

Aceptado: 13/08/2020

Cómo citar este artículo

González-Sánchez AL, Moreno-Monsiváis MG, Cheverría-Rivera S. Cuidado de enfermería perdido percibido por madres de pacientes pediátricos hospitalizados. SANUS. 2020; (15):1-12. [Acceso $\underline{\mathrm{dd}} \underline{\mathrm{mm}} \underline{\text { aal; }}$; Disponible en: $\underline{\text { URL/DOI. }}$ 


\title{
Missed nursing care perceived by mothers of hospitalized pediatric patients
}

\begin{abstract}
Introduction: Missed nursing care is defined as the care required by the patient that is omitted (partially or totally) or significantly delayed. In the pediatric population, it is relevant to identify the missing care given the vulnerability of children, who may not be able to express or demand the necessary attention. Objective: Identify the missed nursing care and its relationship with the reasons perceived by the mothers. Methodology: Descriptive correlational study. One hundred sixty two (162) mothers of pediatric patients from a public hospital participated. Data were collected with the MISSCARE instrument; for the analysis, descriptive and inferential statistics were used. Results: Main misscare situations are planning of discharge and patient education, followed by basic care such as helping, or carrying, mouth and hands washing (82.1\%), checking that the food is at the proper temperature (62.1\%) and bringing the food to the patient as well as check the diet before they eat it (61.1\%). Lack of enough human resources is the main reason why care is missed. Additionally, unexpected increase in the amount of patients and/or workload (47\%) and emergency situations that demand more attention and, thus, generate insufficient time to attend other patients (34.5\%) stood out. Conclusions: Missed care was identified in the hospitalized pediatric patients and is mainly attributed to lack of human resources. This information shall be considered as relevant by the managers in order to strengthen nursing care in pediatric population.
\end{abstract}

Keywords: Nursing; care; Patient; Mother (DeCS). 


\title{
Ausência de cuidados de enfermagem percebidos pelas mães de pacientes pediátricos hospitalizados
}

\begin{abstract}
ABSTRATO
Introdução: O cuidado de enfermagem omitido é definido como o cuidado exigido pelo paciente que é omitido (parcial ou totalmente) ou significativamente atrasado. Na população pediátrica, é relevante identificar a falta de cuidado dada a vulnerabilidade da criança, que pode não conseguir expressar ou exigir a atenção necessária. Objetivo: Identificar os cuidados de enfermagem omitidos e sua relação com os motivos percebidos pelas mães. Metodologia: Estudo descritivo correlacional. Cento e sessenta e duas (162) mães de pacientes pediátricos de um hospital público participaram. Os dados foram coletados com o instrumento MISSCARE; para a análise, utilizou-se a estatística descritiva e inferencial. Resultados: As principais situações de incumprimento são o planejamento da alta e a educação do paciente, seguidos de cuidados básicos como ajudar ou transportar a lavagem da boca e das mãos $(82,1 \%)$, verificar se o alimento está na temperatura adequada $(62,1 \%)$ e levar o alimento ao paciente, bem como verificar a dieta antes de ingeri-la (61,1\%). A falta de recursos humanos suficientes é a principal razão pela qual os cuidados são perdidos. Além disso, destacaram-se o aumento inesperado na quantidade de pacientes e / ou carga de trabalho (47\%) e situações de emergência que demandam mais atenção e, portanto, geram tempo insuficiente para atender outros pacientes (34,5\%). Conclusões: A perda de atendimento foi identificada nos pacientes pediátricos hospitalizados e é atribuída principalmente à falta de recursos humanos. Essas informações devem ser consideradas relevantes pelos gestores para fortalecer a assistência de enfermagem à população pediátrica.
\end{abstract}

Palavras-chave: Enfermagem; Cuidado; Paciente; Mãe (DeCS). 


\section{INTRODUCCIÓN}

La calidad y la seguridad del paciente son fundamentales en la salud ${ }^{1}$; sin embargo, se pueden ver afectadas por errores de comisión y de omisión". "Los errores que se comenten pueden ser un acto de comisión (hacer algo mal como administrar un medicamento de manera incorrecta), o un acto de omisión (no realizar un cuidado o retrasarlo), ambos conducen a un resultado adverso. Los errores de omisión representan un problema mayor debido a que son más difíciles de reconocer que los de comisión" ${ }^{\prime \prime}$. El cuidado de enfermería perdido, se considera un error de omisión y conlleva a resultados negativos que afectan la calidad e incrementan los días de hospitalización y los costos en la atención ${ }^{4}$.

El cuidado perdido ha sido principalmente abordado de acuerdo a la percepción de profesionales enfermería que brindan cuidado a pacientes adultos hospitalizados, existen pocos estudios donde se considera la percepción de los usuarios como receptores del cuidado y estos se han centrado también en población adulta. El acercamiento a la población pediátrica es limitado y se considera relevante. En este sentido, los padres son los tomadores de decisiones legales en los pacientes pediátricos, responsables de evaluar la calidad de la atención prestada a sus hijos, en donde sus opiniones parecen reflejar puntos de vista y actitudes del niño ${ }^{5}$. Un aporte importante de este estudio es incluir la percepción de las madres por ser la voz de sus pacientes en el sistema de salud $y$, por consiguiente, ser quienes representan a los niños. Además, son las personas que permanecen la mayor parte del tiempo de hospitalización junto a sus hijos ${ }^{6}$. El presente estudio de investigación aborda el cuidado de enfermería perdido en pacientes pediátricos, grupo de edad que se considera relevante por ser lábiles a diversos problemas de salud propios de su edad, los cuales son prevenibles y tratados en el mayor de los casos ${ }^{7}$. El Modelo de Cuidado de Enfermería Perdido de Kalisch ${ }^{8}$, se consideró apropiado como soporte teórico del estudio, examina los tres conceptos principales del Modelo de Calidad de Donabedian (estructura, proceso y resultado) ${ }^{9}$.
La estructura se refiere a la infraestructura del hospital, las características de la unidad de atención al paciente y características individuales de enfermería. El proceso es el cuidado de enfermería perdido y el resultado son los efectos directos de la atención de enfermería hacia los pacientes.

Kalisch ${ }^{8}$ incluye en el proceso cuatro tipos de intervenciones de enfermería: (a) Las intervenciones de necesidades individuales se refieren a todas aquellas actividades que se realizan para brindar autocuidado y soporte emocional, orientadas en tratar respuestas humanas en situaciones con afectaciones a la salud, como aplicar medicamentos indicados y responder en menos de 5 minutos al llamado del paciente ${ }^{3,4}$. (b) La planificación del alta y educación al paciente, corresponde a la orientación dada al usuario y a su familiar de manera continua y personalizada, esta debe iniciar desde el momento del ingreso de los pacientes a la unidad hospitalaria y durante toda la estancia, para que al egreso se refuercen las medidas necesarias para la continuidad del cuidado en el domicilio con información suficiente ofrecida al paciente $y$ sus cuidadores $^{10}$. (c) Las intervenciones de cuidado básico se brindan cuando el paciente no tiene autonomía para satisfacer sus necesidades básicas, a estas actividades se les considera rutinarias en la mayor parte de los hospitales, por ejemplo, ayudar a deambular al paciente y cambiar su posición acorde a las necesidades ${ }^{11}$. (d) Intervenciones de cuidado con evaluaciones continuas es estar constantemente valorando al paciente y tomar decisiones adecuadas cuando las condiciones de salud cambian ${ }^{3}$.

Kalisch señala que existen razones por las que el cuidado se pierde, se relacionan con la cantidad de recursos humanos, disponibilidad de recursos materiales, así como las relaciones interpersonales y la transferencia de información ${ }^{4}$, estas incluyen factores de "recursos humanos", "materiales" y de "comunicación". Las razones de acuerdo a los recursos humanos corresponden a las características del personal, la dotación, horarios laborales, experiencia y nivel de competencia en la profesión ${ }^{3,4}$. Las razones atribuidas a los recursos materiales se refieren a 
la no disponibilidad de todo lo necesario para la atención como medicamentos, equipo médico o materiales ${ }^{4}$. Las razones de comunicación son las relaciones interpersonales con el equipo multidisciplinario para tener una mejor planeación de los cuidados requeridos ${ }^{3,4}$.

El cuidado de enfermería que se brinda a la población pediátrica es crucial para el cumplimiento de estándares e indicadores de calidad acordes a esta población, es un fenómeno importante que debe ser medido al interior de las organizaciones de salud debido a su vinculación con los resultados adversos evitables de los pacientes. En consideración a lo anterior, el objetivo del estudio fue identificar el cuidado de enfermería perdido y su relación con los recursos humanos, materiales y de comunicación en función de la percepción de las madres de pacientes pediátricos hospitalizados.

\section{METODOLOGÍA}

El estudio fue descriptivo y correlacional, participaron madres de pacientes pediátricos de un hospital público de tercer nivel ubicado en la ciudad de León Guanajuato, México, en el periodo de diciembre del 2018 a marzo del 2019. La muestra se estimó utilizando el paquete n-Query Advisor, versión 4.0, mediante análisis de correlación con una significancia de 0.05 , correlación esperada de 0.40 y potencia de $90 \%$, dando una muestra total de 162 madres. El muestreo fue probabilístico sistemático de 1 en 2 , a través de un listado de pacientes pediátricos hospitalizados, se seleccionó de manera aleatoria a la primera madre y posteriormente de manera sistemática de acuerdo al número de inicio.

Los criterios de inclusión fueron: madres que asumieron el rol de cuidador principal de sus hijos hospitalizados, con un tiempo igual o superior a 72 horas de internamiento. Se excluyeron del estudio a las madres que durante la recolección de datos sus hijos se encontraron en hospitalización pediátrica con cuidados avanzados o especializados de terapia intensiva y que se encontraron allí por no haber disponibilidad de cama en áreas de terapia intensiva

Previo a la recolección, a las madres seleccionadas se les explicó el objetivo de la investigación y se solicitó su participación voluntaria, así como firmar el consentimiento informado. Posteriormente se procedió a aplicar el instrumento a manera de entrevista. Se cuidó que la aplicación se realizara en un momento en el que no interfiriera con los cuidados de apoyo que las madres realizan a sus hijos.

El cuidado de enfermería perdido se midió con el instrumento MISSCARE ${ }^{12}$ los reactivos originales se adaptaron a las madres cuidando en todo momento mantener la esencia de cada uno (ejemplo: el reactivo 1 señala: "con qué frecuencia ayuda al paciente a deambular durante el día de acuerdo a su indicación" se adaptó de la siguiente manera: "Con qué frecuencia el personal de enfermería asiste a su hijo (a) en la deambulación según la indicación"). El instrumento contiene 48 preguntas correspondientes a tres secciones, la primera permitió perfilar a las madres mediante 9 preguntas, 4 relacionadas con características de las madres (edad, sexo, escolaridad y número de hijos) y 5 con datos del paciente pediátrico (edad, sexo, lugar que ocupa en la familia, hospitalizaciones previas y número de días hospitalizado). La segunda sección midió el cuidado de enfermería perdido cuenta con un total de 24 preguntas (Alfa de Cronbach 0.85), divididos en cuatro dimensiones: intervenciones de necesidades individuales, planificación del alta y educación al paciente, intervenciones de cuidado básico e intervenciones de cuidado con evaluaciones continuas; las respuestas estuvieron compuestas por una escala Likert con puntuaciones de 0 a 5 , donde 0 corresponde a lo desconozco y 5 siempre se pierde.

La opción "lo desconozco" se agregó para calificar todos aquellos aspectos de la atención de enfermería que las madres fueran incapaces de identificar. Las respuestas se convirtieron en una escala dicotómica, en donde 1, 2 y 3 se consideraron como cuidado otorgado, 4 y 5 cuidado perdido.

La tercera sección mide las razones para el cuidado de enfermería perdido con 15 
preguntas (Alfa de Cronbach 0.71), que se clasifican en recursos humanos, materiales y de comunicación. Las respuestas corresponden a una escala Likert con opciones de 4 razón significativa a 1 no es razón y 0 lo desconozco. La información obtenida se capturó en una base de datos en el paquete estadístico Statistical Package for the Social Sciences, Versión 25 (SPSS V25.0). Se utilizó estadística descriptiva y análisis de correlación de Pearson por la normalidad de los datos obtenidos de acuerdo a la prueba de kolmogorov Smirnov. La información se agrupó para analizar los cuidados perdidos y no perdidos con apoyo de un índice global con valores de 0 a 100 para cada dimensión de cuidado, así mismo para las razones del cuidado perdido.

La investigación fue aprobada por el Comité de Ética e Investigación de la Facultad de Enfermería y Nutrición de la Universidad Autónoma de San Luis Potosí, México con registro CEIFE--2018-274, así como por el Comité de Investigación del Hospital Regional de Alta Especialidad del Bajío con el número de registro Cl/HRAEB/2018/032. Durante la recolección se cuidó el respeto a los derechos, bienestar y dignidad de las madres, cumpliendo con lo dispuesto en la Norma Oficial Mexicana en materia de Investigación ${ }^{13} .4$

\section{RESULTADOS}

La edad de las madres se ubicó en una media de 33.9 años $(D E=8.7)$, en cuanto al nivel de estudios predominó la secundaria completa (40.7\%). La media del número de hijos fue de 2.9 $(D E=1.9)$, la edad de los hijos fue de 6.9 años $(D E=5.5)$ en promedio, predominó el sexo masculino.

La tabla 1 muestra el índice global y por dimensiones del cuidado de enfermería perdido percibido por las madres. En la dimensión de intervenciones de necesidades individuales se identificó que el cuidado que más se pierde se atribuye a la presencia de la enfermera en las visitas del cuidado interdisciplinario (41.9\%), seguido de la ayuda a su hijo por el personal de enfermería con las necesidades de ir al baño
(38.9\%). El cuidado otorgado fue para la administración de medicamentos con apego al horario programado (97.5\%), apoyo emocional por parte del personal de enfermería y evaluación de los medicamentos que le administraron a sus hijos, ambos con un $94.4 \%$. En relación a la dimensión de la planificación del alta y educación al paciente, los cuidados que más se pierden corresponden a que "el personal de enfermería no le enseña cómo realizar los cuidados a su hijo (a) en el hogar" (42.6\%), seguido de no informar acerca de la enfermedad de su hijo (37.1\%).

En la dimensión de cuidado básico el cuidado perdido lo observaron en actividades como lavado bucal a su hijo (a) y lavado de manos de su hijo (a), ambos con $82.1 \%$, verificar que la comida de su hijo (a) esté a una temperatura tolerable o adecuada (62.3\%) y acercar y verificar la dieta antes de que la coma su hijo (a) (61.1\%). Los cuidados otorgados que se realizan principalmente fueron que enfermería contribuye al cuidado de la piel, lesiones o heridas de su hijo (a) (71.6\%) y que el personal de enfermería le ayuda o realiza el baño a su hijo (a) $(63.5 \%)$.

En la dimensión de intervenciones de cuidado con evaluaciones continuas el cuidado que más se perdió fue la monitorización por parte del personal de enfermería de los exámenes indicados (11.2\%). Las madres perciben que no hay omisiones de cuidado en la medición los signos vitales y en la verificación de la cantidad de líquidos que toma o que les administran a sus hijos (100\%).

Las intervenciones con mayor cuidado perdido se identificaron en la "planificación del alta y educación al paciente" (Media $=58.2 ; D E=28.7$ ), seguido de "cuidado básico" (Media = 55.6; $D E=17.6)$. La menor omisión fue para las "intervenciones de cuidado con evaluaciones continuas" (Media $=26.2 ; D E=8.8$ ). La media del puntaje global de cuidado perdido se ubicó en $42(D E=11.6)$.

La Tabla 2 muestra los índices de razones para el cuidado perdido percibidas por las madres. $\mathrm{Se}$ atribuyen en primer orden al recurso humano (Media $=41.1 ; D E=16$ ), seguido de los recursos materiales y de comunicación. 
Tabla 1. Índices por dimensión y global del cuidado de enfermería perdido percibido por las madres de pacientes de un Hospital Público del Estado de Guanajuato, México, 2019.

\begin{tabular}{lcccc}
\hline \multicolumn{1}{c}{ Índices } & Media & $\begin{array}{c}\text { Desviación } \\
\text { Estándar }\end{array}$ & & \multicolumn{2}{c}{\begin{tabular}{c} 
Intervalo de confianza \\
\cline { 4 - 5 }
\end{tabular}} & & & $\begin{array}{c}\text { Límite } \\
\text { Inferior }\end{array}$ & $\begin{array}{c}\text { Límite } \\
\text { Superior }\end{array}$ \\
\hline $\begin{array}{l}\text { Global de cuidado de enfermería } \\
\text { perdido }\end{array}$ & 42.0 & 11.6 & 40.1 & 43.8 \\
$\begin{array}{l}\text { Intervenciones de necesidades } \\
\text { individuales }\end{array}$ & 37.6 & 12.8 & 35.6 & 39.6 \\
$\begin{array}{l}\text { Planificación del alta y educación al } \\
\text { paciente }\end{array}$ & 58.2 & 28.7 & 53.8 & 62.7 \\
$\begin{array}{l}\text { Intervenciones de cuidado básico } \\
\begin{array}{l}\text { Intervenciones de cuidado con } \\
\text { evaluaciones continuas }\end{array}\end{array}$ & 55.6 & 17.6 & 52.8 & 58.3 \\
\hline
\end{tabular}

En cuanto a las razones atribuidas a los recursos humanos, las madres identificaron como razón significativa a moderada para el cuidado de enfermería perdido el incremento de pacientes en el servicio (47\%) y las situaciones de urgencia de los pacientes del área o servicio que a las enfermeras les demandan mayor atención y les queda poco tiempo para atender a otros pacientes (34.5\%).

Para las razones vinculadas con los recursos materiales, las madres identificaron que los medicamentos, materiales y equipos no están disponibles cuando se requieren $(27.7 \%$ y $17.3 \%$ respectivamente) y materiales/equipos no funcionan correctamente con un $14.2 \%$.

Respecto a la comunicación, las madres perciben como razones significativas a moderadas para el cuidado perdido las tensiones o fallas en la comunicación con otros departamentos (17.3\%) y el que otros departamentos no proporcionen los cuidados necesarios (14.2\%).

Para estimar la vinculación en las variables del estudio se utilizó la correlación de Pearson por la normalidad de los datos obtenidos. Se obtuvo una correlación positiva significativa entre las razones de recursos humanos, materiales y de comunicación con todas las dimensiones de cuidado (Tabla 3).

\section{DISCUSIÓN}

El estudio permitió identificar el cuidado perdido percibido por las madres de pacientes pediátricos hospitalizados. Esto es importante de considerar en las organizaciones debido a que está documentado que una gran cantidad de eventos adversos son consecuencia de la atención de enfermería que se retrasa u omite; 
Tabla 2. Índices por dimensión de razones que contribuyen al cuidado de enfermería perdido percibido por las madres de pacientes de un Hospital Público del Estado de Guanajuato, México, 2019

\begin{tabular}{|c|c|c|c|c|}
\hline \multirow[b]{2}{*}{ Índices } & \multirow[b]{2}{*}{ Media } & \multirow[b]{2}{*}{ Desviación Estándar } & \multicolumn{2}{|c|}{ Intervalo de confianza $95 \%$} \\
\hline & & & Límite Inferior & Límite Superior \\
\hline Recursos Humanos & 41.1 & 16.0 & 38.6 & 43.5 \\
\hline Recursos Materiales & 39.4 & 19.9 & 36.3 & 42.5 \\
\hline Comunicación & 34.2 & 14.2 & 31.9 & 36.4 \\
\hline
\end{tabular}

Fuente: Encuesta MISSCARE para madres de pacientes hospitalizados.

$n=162$

Tabla 3. Coeficientes de correlación entre las dimensiones de cuidado y las razones para que se pierda el cuidado, percibido por las madres de los pacientes de un Hospital Público del Estado de Guanajuato, México, 2019

\section{Razones para el cuidado de enfermería perdido}

\begin{tabular}{lccc} 
Dimensiones de cuidado & Recursos humanos & $\begin{array}{c}\text { Recursos } \\
\text { Materiales }\end{array}$ & Comunicación \\
\hline $\begin{array}{l}\text { Global de cuidado de enfermería } \\
\text { perdido }\end{array}$ & $0.329+$ & $0.272+$ & $0.347+$ \\
$\begin{array}{l}\text { Intervenciones de necesidades } \\
\text { individuales }\end{array}$ & $0.345^{+}$ & $0.227+$ & $0.366^{+}$ \\
$\begin{array}{l}\text { Planificación del alta y educación } \\
\text { al paciente }\end{array}$ & $0.164^{*}$ & $0.199^{*}$ & $0.203+$ \\
$\begin{array}{l}\text { Intervenciones de cuidado básico } \\
\text { Intervenciones de cuidado con }\end{array}$ & $0.247+$ & $0.208+$ & $0.279+$ \\
evaluaciones continuas & $0.270+$ & $0.242+$ & $0.213+$
\end{tabular}

${ }^{*} \mathrm{p}<0.05 ;+\mathrm{p}<0.01$ 
así mismo, se ha asociado con mayor estancia, costos y reingresos hospitalarios, así como con complicaciones en el paciente ${ }^{4,14}$.

La población pediátrica debe ser una prioridad en la atención ${ }^{7}$ debido a que este grupo de edad se considera vulnerable a diversos problemas de salud y pueden no manifestar necesidades de cuidado que afectan sus resultados, por lo que el apego a las intervenciones de cuidado reduce la probabilidad de complicaciones 5 .

Las madres percibieron cuidado perdido en mayor proporción en las "intervenciones de planificación del alta y educación al paciente", estas señalaron escasa enseñanza de cómo realizar los cuidados a sus hijos al alta hospitalaria. Lo anterior se pudiera atribuir a que al interior de las instituciones de salud es el personal médico el que tradicionalmente da dichas indicaciones; sin embargo, existe un escaso involucramiento del profesional de enfermería en el rol de educador, lo cual representa un área de oportunidad debido a que es el personal de salud que permanece más tiempo junto al paciente, por lo que se considera es el profesional idóneo para identificar las necesidades de cuidado y proporcionar la educación a los padres o cuidadores durante la estancia hospitalaria como una actividad necesaria para empoderarlos para el cuidado en el hogar, producto del trabajo en equipo entre los padres y enfermería ${ }^{14}$.

En segundo orden refirieron cuidado perdido en la dimensión de "intervenciones de cuidado básico", el cuidado con mayor omisión correspondió a ayudar o realizar el lavado bucal y el lavado de manos a sus hijos, verificar que la comida esté a una temperatura tolerable o adecuada y acercar la comida y verificar la dieta antes de que la coman sus hijos. De acuerdo a la literatura, es común identificar cuidado perdido en las intervenciones de cuidado básico, estas omisiones pueden atribuirse a que los profesionales de enfermería tienden a no priorizar estas actividades por ser cuidados poco complejos, además porque algunas veces los pacientes las realizan por ellos mismos o con ayuda de familiares ${ }^{15,16}$. En el caso de las madres son ellas quienes la mayoría de las veces realizan este tipo de atenciones.

Estos hallazgos concuerdan con lo reportado en un estudio ${ }^{17}$, donde se identificó al entrevistar a pacientes adultos hospitalizados, que la "planificación del alta y educación al paciente" y el "cuidado básico" son los principales cuidados que se pierden durante la estancia hospitalaria. Las madres consideraron que el recurso humano es la principal razón significativa para el cuidado perdido. Señalaron que el aumento en el número de pacientes, la carga de trabajo del área y las situaciones de urgencia de los pacientes del servicio le demanda al personal de enfermería mayor atención y les queda poco tiempo para atender a otros pacientes. Lo antes descrito coincide con una investigación ${ }^{17}$, donde se observaron razones similares para el cuidado perdido en pacientes adultos hospitalizados. Así mismo, concuerda con los hallazgos de un estudio en el $2017^{15}$ de cuidado de enfermería perdido en profesionales de enfermería que brindaron atención a población adulta.

Las madres atribuyen en segundo orden el cuidado perdido a las razones vinculadas con los recursos materiales como la falta de disponibilidad de los medicamentos cuando se necesitan y finalmente a los recursos de comunicación. Este aspecto des relevante para los administradores de enfermería y demanda buscar estrategias que favorezcan que el personal cuente con los insumos necesarios cuando se les requiere para proporcionar el cuidado de acuerdo a las necesidades de los pacientes. Estos resultados son congruentes a lo reportado por otros autores ${ }^{17,18}$ quienes mencionan que los recursos humanos, materiales y de comunicación se relacionaron positiva y significativamente con el cuidado perdido.

Existe escasa evidencia de estudios de investigación que aborden la temática del cuidado perdido en población pediátrica hospitalizada. Sin embargo, algunos estudios donde se considera la opinión de los padres son consistentes al señalar que la calidad de la atención depende de aspectos relacionados con evaluaciones continuas y explicaciones acerca del cuidado hospitalario y en el hogar. Es 
importante que la atención de enfermería responda a las expectativas de los padres de los pacientes pediátricos hospitalizados ${ }^{19}$ por ser un indicador de calidad y seguridad en la entrega del cuidado.

\section{CONCLUSIONES}

El cuidado perdido afecta la calidad y la seguridad del paciente por lo que es relevante identificar las intervenciones de cuidado y las razones vinculadas con las omisiones en la atención. Los hallazgos del presente estudio permiten contar con información relevante para los gestores de enfermería que les facilite desarrollar e implementar estrategias de mejora en la atención a este grupo poblacional. Sin embargo, es importante en futuros estudios considerar la participación de cuidadores de pacientes pediátricos de diferentes instituciones de salud del ámbito público y privado que permitan tener una visión más amplia que apoye la toma de decisiones orientadas a fortalecer el cuidado de enfermería en la población pediátrica hospitalizada.

\section{CONFLICTO DE INTERESES}

Los autores declaran no tener ningún conflicto de intereses.

\section{FINANCIAMIENTO}

El estudio se financió con la beca de maestría otorgada a la MAE. Ada Lizette González Sánchez por parte del Consejo Nacional de Ciencia y Tecnología (CONACYT). Publicación financiada con recursos PROFEXCE 2020

\section{REFERENCIAS BIBLIOGRÁFICAS}

1. Organización Mundial de la Salud (OMS). Más que palabras. Marco Conceptual de la Clasificación Internacional para la Seguridad del Paciente. Informe técnico definitivo [Internet]. OMS. Enero 2009. [consultado Mayo 2018]. Disponible en: https://www.who.int/patientsafety/implementation/icps/icps full report es.pdf

2. Kalisch B. Missed nursing care: a qualitative study. J Nurs Care Qual [Internet]. 2006 [consultado Enero 2019]; 21 (4): 306-313. Disponible en: http://southbaynursingqsen.pbworks.com/f/Missed+Care.pdf

3. Kalisch B, Xie B. Errors of Omission: Missed Nursing Care. West J Nurs Res [en línea]. 2014 [consultado Agosto 2019]; 1(16) 1-16. Disponible en:

http://wjn.sagepub.com/content/early/2014/04/28/0193945914531859 doi: 10.1177/0193945914531859

4. Kalisch B, Landstrom G, Williams R. Missed nursing care: Errors of omission. Nurs Outlook [Internet]. 2009 [consultado marzo 2019]; 57(1): 3-9. Disponible en:

https://www.ncbi.nlm.nih.gov/pubmed/19150261 doi: 10.1016/j.outlook.2008.05.007.

5. Salmani N, Hasanvand Sh, Bagheri I, Mandegari Z. Nursing care behaviors perceived by parents of hospitalized children: qualitative study. Int J Pediatr [Internet]. 2017 [consultado Febrero 2019]; 5(7): 5379-89. Disponible en:

https://www.researchgate.net/publication/318094940 Nursing Care Behaviors Perceived by P arents of Hospitalized Children A Qualitative Study doi: 10.22038 /ijp.2017.23123.1940

6. Quero A. Los cuidados no profesionales en el hospital: la mujer cuidadora. Enfría Clínica. 2003; 13(6):348-356. doi: 10.1016/S1130-8621(03)73835-2 
7. Lake B. Cordova P, Barton S, Singh S, Agosto P, Beth E, et al. Missed Nursing Care in Pediatrics. Hosp Pediatr [Internet]. 2017 [consultado Junio 2018]; 7(7). Disponible en: https://www.ncbi.nlm.nih.gov/pubmed/28611146 doi: https://doi.org/10.1542/hpeds.2016-0141

8. Kalisch B, Landstrom G, Hinshaw A. Missed nursing care: A concept analysis. J Adv Nurs [Internet]. 2009 [consultado Noviembre 2018]; 65(7): 1509-1517. Disponible en: https://www.researchgate.net/publication/24441229 Missed Nursing Care A Concept Analysi $\underline{\mathrm{s}}$

doi: 10.1111/j.1365-2648.2009.05027.x

9. Donabedian A. The quality of care how can it be assessed?. JAMA [Internet]. 1988 [consultado marzo 2019]; 260(12): 1743-1748. Disponible en:

https://www.ncbi.nlm.nih.gov/pubmed/3045356 doi: 10.1001/jama.260.12.1743

10. Navarrete S. Capítulo 8. Educación al paciente y a su familia. Rev. Colombiana de Cardiología. 2016 [consultado 23 de mayo del 2018]; 23(S1):34-37. Disponible en:

https://www.sciencedirect.com/science/article/pii/S0120563316000140

11. Bittner NP, Gravlin G. Critical thinking, delegation, and missed care in nursing practice. J Nurs Admin [Internet]. 2009 [Consultado el 25 de febrero 2019]; 39(3): 142-146.

DOI 10.1097/NNA.0b013e31819894b7

12. Kalisch BJ, Williams RA. Development and psychometric testing of a tool to measure missed nursing care. J Nurs Admin [Internet]. 2009[Consultado Septiembre 2018]; 39(5): 211-219. Disponible en: https://www.ncbi.nlm.nih.gov/pubmed/19423986

doi: 10.1097/NNA.0b013e3181a23cf5

13. Norma Oficial Mexicana NOM-012-SSA3-2012. Establece los criterios para la ejecución de proyectos de investigación para la salud en seres humanos [en línea]. Diario Oficial de la Federación. 4 enero 2013. Secretaria de Salud. [consultado 2 de marzo de 2019]. Disponible en: http://dof.gob.mx/nota detalle.php?codigo $=5284148 \&$ fecha $=04 / 01 / 2013$

14. Alvarado $H$, Rodríguez $S$. Necesidades percibidas de atención por niños, cuidadores y enfermeros durante la hospitalización en una unidad de cuidado intensivo. Investig Enferm. Imagen Desarr [Internet]. 2015 [consultado Enero 2019]; 17(1):113-130. Disponible en: https://doi.org/10.11144/Javeriana.IE17-1.npan

15. Hernández-Cruz R, Moreno-Monsiváis MG, Cheverria-Rivera S, Díaz Oviedo A. Factores que influyen en el cuidado de enfermería perdido en pacientes de un hospital privado. Rev. LatinoAm. Enfermagen [Internet]. 2017 [consultado Noviembre 2018]; 25:e2877. Disponible en: http://www.scielo.br/pdf/rlae/v25/es 0104-1169-rlae-25-e2877.pdf doi: http://dx.doi.org/10.1590/1518-8345.1227.2877

16. Kalisch B, Lee K. Missed nursing care: Magnet versus non-Magnet hospitals. J Nurs Outlook [Internet]. 2012 [Consultado Enero 2019]; 60(5):32-39. Disponible en: https://www.ncbi.nIm.nih.gov/pubmed/22824471 doi: 10.1016/j.outlook.2012.04.006

17. Moreno-Monsiváis $M$, Moreno-Rodríguez $C$, Interial-Guzmán M. Missed Nursing Care in Hospitalized Patients. Aquichan [Internet]. 2015 [consultado Diciembre 2018]; 15(3):318-328. Disponible en: http://www.scielo.org.co/scielo.php?script=sci arttext\&pid =S1657-59972015000300002 doi: 10.5294/aqui.2015.15.3.2

18. Kalisch BJ, Tschannen $D$, Lee $H$, Friese CR. Hospital variation in missed nursing care. Am J Med Qual [Internet]. 2011[consultado Enero 2019]; 26(4): 291-299. Disponible en: https://www.ncbi.nlm.nih.gov/pubmed/21642601 doi:10.1177/1062860610395929 
19. González del Águila N, Romero Quechol G. Dimensiones de la calidad del cuidado de enfermería y satisfacción del padre o tutor del paciente pediátrico hospitalizado. Rev. Enferm IMSS [internet]. 2017 [consultado Marzo 2019]; 25(3):173-180. Disponible en:

https://www.medigraphic.com/cgi-bin/new/resumen.cgi?IDARTICULO $=73563$

Copyright (C) 2020 SANUS

Artículo de acceso abierto distribuido bajo Licencia Creative Commons 